\title{
Mathematical Modelling of Subdiffusion-reaction Systems
}

\begin{abstract}
A.A. Nepomnyashchy *
Department of Mathematics, Technion - Israel Institute of Technology, 32000 Haifa, Israel

Abstract. A review of recent developments in the theoretical description and mathematical modelling of subdiffusion-reaction systems is presented. A number of subdiffusion-reaction models are discussed, including models of subdiffusion-limited reactions and models with intertwined subdiffusion and reaction operators. Specific features of the instability development and pattern formation are discussed. Some applications of subdiffusion-reaction models to specific natural problems are mentioned.
\end{abstract}

Keywords and phrases: subdiffusion, reactions, fractional derivatives, patterns

Mathematics Subject Classification: 35K57, 35R11, 60J60, 82C70

\section{Introduction}

The phenomenon of anomalous diffusion attracted much attention of researchers [1] - [4]. The basic manifestation of this phenomenon is an unusual scaling of the mean square displacement (MSD) of the random walk with time: $\left\langle(\Delta \mathbf{r})^{2}\right\rangle \sim t^{\gamma}, \gamma \neq 1$, which is incompatible with normal diffusion properties and apparently contradicts the central limit theorem [5]. For $\gamma<1(\gamma>1)$ the diffusion process is called "subdiffusion" ("superdiffusion").

The reasons for anomalous diffusion are diverse. A typical explanation of this phenomenon, which can be given in the framework of the continuous time random walk (CTRW) model [3], is the existence of "fat tails" in the probability distribution function (pdf) of the random walk, which causes divergence of low order moments and hence the violation of the validity conditions of the central limit theorem. Specifically, for a long-tail waiting time pdf leading to the divergence of the characteristic waiting time, the normal diffusion equation is replaced by an integro-differential equation containing fractional derivatives in time, which provides a subdiffusive anomalous scaling. The random walk with a Lévy distribution [6] of the jump length pdf, which is characterized by a divergent jump length variance, can be described on a large scale by a diffusion equation with a fractional power of the Laplacian, which gives rise to a superdiffusive anomalous scaling (for moments which converge).

However, there exist alternative explanations of the phenomenon of the anomalous diffusion. For instance, the same subdiffusive anomalous law for a MSD can be observed also for a fractional Brownian motion (FBM) which is a Gaussian process [7]. It can be driven by the action of an external Gaussian

*Corresponding author. E-mail: nepom@math.technion.ac.il 
noise $\xi(t)$ with a slowly decreasing negative autocorrelation function, $\left\langle\xi(t) \xi\left(t^{\prime}\right)\right\rangle \sim-\left|t-t^{\prime}\right|^{\gamma-2}$ (fractional Gaussian noise), or by an internal fractional Gaussian noise, if the motion of a particle is characterized by a power-law memory for the friction, due to the fluctuation-dissipation theorem (see, e.g., [8] - [10] and the references therein). A random walk on fractal sets (RWF) and in disordered systems also leads to an anomalous diffusion [1], [2]. A mixed origin of subdiffusion is also possible [11]. Moreover, even the knowledge of the probability distribution function is not sufficient for recovering the underlying stochastic nature of the process leading to subdiffusion: identical pdfs have been revealed for the random walk on a comb (nonstationary model which belongs to the CTRW class) and the random walk on the random walk (stationary ergodic model) [12].

A comprehensive description of physical scenarios leading to subdiffusion and the tools for distinguishing between those scenarios are described in the review paper [13]. Checking the ergodicity properties of the process is the primary test for the clarification of the underlying mechanism of the anomalous behavior: the CTRW is non-ergodic, while FBM and RWF are ergodic. The computation of the step autocorrelation function allows to distinguish between the pure CTRW process (with uncorrelated steps) and other mechanisms of subdiffusion (FBM and RWF) which demonstrate anticorrelated steps. An additional test has been suggested for distinguishing between the FBM and RWF mechanisms of subdiffusion [14].

The description of reactions which take place in systems with anomalous diffusion is an even more difficult task. One can distinguish between a direct consideration of the molecular motion and reactions between molecules (microscopic approach) and the derivation of equations characterizing the large-scale behavior of continuous functions, e.g., probability distribution functions and densities (macroscopic approach). The present review paper is devoted to the applications of the latter approach. Construction of a mathematical model incorporating reactions is straightforward for superdiffusive systems where diffusion is characterized by a spatial non-locality but no memory is present [15], [16]. In the case of subdiffusion (systems with memory), the situation is much more intricate. It has been shown by Henry et al. that a "naive" addition of a reaction term into the evolution equation with a reaction rate independent of the diffusion is physically inconsistent and may lead to unphysical negative values of the probability distribution function [17]. As a matter of fact, there is no universal model for reactions in a subdiffusive system; the description depends on the details of the underlying physics. Generally, there are two main groups of macroscopic models. In the first group of models, appropriate for diffusion-limited kinetics, the diffusion and reaction terms appear in the equations in an additive way, but the time evolution is described by fractional order derivatives, i.e., the memory kernel is the same for the diffusion and reaction terms [18] [20]. The other group of models appropriate for activation-limited reactions, is based on the assumption that the reaction constants are unaffected by the diffusion, but the change of the chemical composition due to the reaction affects the diffusion process. Thus, the diffusion and the reaction are intertwined [21]. In the case of a linear reaction a closed system of fractional PDEs governing concentrations of species can be constructed [21], [22]. In the case of a nonlinear reaction, the use of two temporal variables, the actual time and the time elapsed since the last jump (the "age" of the particle) is convenient [23] - [26]. A somewhat controversial point is the age prescribed to a particle after the reaction event. In [23], [24] the particle is "rejuvenated" after it reacts, i.e., its waiting time distribution is set as if the particle has performed a jump. In [21], [27], [28], [25] it is assumed that the reaction does not change the age of the particle. The consequences of both assumptions are discussed in [29]. The microscopic simulations are in favor of the latter assumption [30], but generally the validity of both assumptions can depend on the particular physical problem.

This review is devoted to the discussion of recent developments in the theoretical description and mathematical modelling of subdiffusion-reaction systems. In Sec. 2, the models for subdiffusion-limited reactions are considered. Sec. 3 contains the description of models for activation-limited reactions. Instabilities and pattern formation in subdiffusion-reaction systems is the subject of Sec. 4. Some applications of subdiffusion-reaction models in particular biological problems are presented in Sec. 5 . 


\section{Models for subdiffusion-limited reactions}

As mentioned in Sec. 1, the models of subdiffusion-reaction systems can be divided into two groups. The first group of models [19], [20], which are appropriate for subdiffusion-limited reactions, have the structure

$$
{ }_{0}^{C} D_{t}^{\gamma} \mathbf{u}=\mathrm{K} \partial_{x}^{2} \mathbf{u}+\mathbf{f}(\mathbf{u}), 0<\gamma<1,
$$

or

$$
\partial_{t} \mathbf{u}={ }_{0} D_{t}^{1-\gamma}\left[K \partial_{x}^{2} \mathbf{u}+\mathbf{f}(\mathbf{u})\right]
$$

where

$$
{ }_{a}^{C} D_{t}^{\gamma} g(t)=\frac{1}{\Gamma(1-\gamma)} \int_{a}^{t} \frac{\partial_{\tau} g(\tau)}{(t-\tau)^{\gamma}} d \tau
$$

is the Caputo fractional derivative, and

$$
{ }_{a} D_{t}^{1-\gamma} g(t)=\frac{1}{\Gamma(\gamma)} \frac{\partial}{\partial t} \int_{a}^{t} \frac{g(\tau)}{(t-\tau)^{1-\gamma}} d \tau
$$

is the Riemann-Liouville fractional derivative, $\mathbf{u}=\left\{u_{i}\right\}, i=1, \ldots, n$ is the vector of molecule densities of different chemical components, $\mathbf{f}=\left\{f\left(u_{i}\right)\right\}, i=1, \ldots, n$ is the vector of reaction rates, and $\mathrm{K}=\left\{K_{i j}\right\}$, $i=1, \ldots, n, j=1, \ldots, n$ is the matrix of diffusion coefficients. For this kind of model, the factors that slow down the diffusion, slow down the reaction simultaneously in the same way. Models of this kind were derived from the CTRW model for the recombination kinetics [19] and instantaneous creation and annihilation processes in subdiffusive media [17]. It was also suggested for the description of results of Monte-Carlo simulations in [20].

Model (2.2) has been applied for the consideration of a transition zone between two initially separated substances $A$ and $B$ reacting according to the formula $m A+n B \rightarrow C$, where $C$ is an inert product. The subdiffusion-reaction process is governed by the system of equations [31]

$$
{ }_{0}^{C} D_{t}^{\gamma} u_{A}=K_{A} \partial_{x}^{2} u_{A}-m R,{ }_{0}^{C} D_{t}^{\gamma} u_{B}=K_{B} \partial_{x}^{2} u_{B}-n R ; R=k u_{A}^{m} u_{B}^{n}
$$

with initial conditions

$$
x<0, u_{A}(x, 0)=u_{0 A}, u_{B}(x, 0)=0 ; x>0, u, u_{A}(x, 0)=0 ; u_{B}(x, 0)=u_{0 B} .
$$

The case $m=n=1$ has been studied by Yuste et al. [20]. In the case of normal diffusion, it is known that the width of the reaction zone grows with time as $W_{r} \sim t^{1 / 6}$, while the width of the depletion zone, where the concentrations of components $A$ and $B$ are small, grows as $W_{d} \sim t^{1 / 2}$. In the case of subdiffusion, it has been found that $W_{r} \sim t^{\gamma / 6}, W_{d} \sim t^{\gamma / 2}$. The center of the reaction zone moves according to the law $x_{r}(t) \sim t^{\gamma / 2}[32]$. The same scaling law has been found in the case of one non-diffusive and one subdiffusive reactant [33]. In the general case, analytical and numerical solutions of $(2.3),(2.4)$ have been obtained in [31].

Note that system (2.2) exhibits long-time memory effects but no ageing. In a bistable system, equation (2.2) predicts the appearance of a reaction front moving with the constant velocity, which depends on the parameter $\gamma$. As an example, let us consider a one-component model with $n=1$. The final shape of the front in the form of a traveling wave solution achieved a long time after the beginning of the process, is obtained by the replacement of the initial time instant by $-\infty$,

$$
{ }_{-\infty}^{C} D_{t}^{\gamma} u=D \partial_{x}^{2} u+f(u)
$$

and substitution of the traveling wave ansatz $u(x, t)=w(z), z=x-c t$ into $(2.5)$. The problem can be solved analytically for a piecewise linear function $f(w)=k[w-H(w-a)]$, where $k>0,0<a<1, H$ is a Heaviside function [34]. An exact analytical expression is obtained for the velocity of the front,

$$
c=\operatorname{sgn}(2 a-1) b^{\frac{1}{\gamma}}\left[\frac{k}{(2-\gamma)\left(\frac{1}{2}-b\right)}\right]^{\frac{1}{\gamma}-\frac{1}{2}}\left[\frac{D}{1-\gamma\left(\frac{1}{2}-b\right)}\right]^{\frac{1}{2}}, b=\left|a-\frac{1}{2}\right| .
$$


Ahead of the front (i.e., for $z>0$ when $c>0$, and for $z<0$ when $c<0$ ) the solution is described by the exact analytical formula

$$
w(z)=\frac{1}{2}(1+\operatorname{sgn}(z))-\operatorname{sgn}(c) \frac{k e^{-Q_{*}|z|}}{2 D Q_{*}^{2}-\gamma\left(|c| Q_{*}\right)^{\gamma}},
$$

where $Q_{*}$ is a solution of the equation

$$
-D Q_{\star}^{2}+k+\left(|c| Q_{\star}\right)^{\gamma}=0,
$$

which is proved to be unique. The tail behind the front is characterized by an algebraic decay,

$$
w(z) \sim \frac{1}{2}(1+\operatorname{sgn}(z))+\frac{1}{2 k \pi}\left|\frac{c}{z}\right|^{\gamma} \Gamma(\gamma) \sin (2 \tilde{\gamma})(\operatorname{sgn}(c)-\operatorname{sgn} z)+o\left(|z|^{-\gamma}\right) .
$$

A more general, but still exactly solvable model with a piecewise linear reaction function,

$$
f(u)=-k_{1} u, 0<u<u_{*} ; f(u)=k_{2}(1-u), u_{*}<u<1,
$$

with $k_{1} \neq k_{2}$, was considered in [36]. That model allows to consider fronts between stable states (in the case $k_{1}>0, k_{2}>0$ ) and fronts between a stable state and an unstable state (in the case $k_{1}<0$, $k_{2}>0$ ). In the former case, a unique value of the front velocity is found. In the latter case, a family of traveling wave solutions ("pulled fronts") is revealed. Also, special "pushed fronts" are possible under definite conditions.

A generalization of the Seki-Lindenberg subdiffusion-reaction model described above has been suggested by Kosztołowicz [35] who has replaced the CTRW characterized by uncorrelated walker's steps with a persistent random walk that is characterized by a definite probability $\beta$ of changing the velocity direction. For a $A+B \rightarrow B$ reaction (species $A$ performs subdiffusion, species $B$ is motionless), starting with a discrete probabilistic model, he has derived the following Cattaneo subdiffusion-reaction equation,

$$
\begin{gathered}
(1-2 \beta) \tau{ }_{0}^{C} D_{t}^{2 \gamma} u+2 \beta{ }_{0}^{C} D_{t}^{\gamma} u=2(1-\beta) K \partial_{x}^{2} u-(1-2 \beta) \tau K \partial_{x}^{2}{ }_{0}^{C} D_{t}^{\gamma} u \\
-R \delta\left(x-x_{r}\right)\left[2 \beta u+(1-2 \beta) \tau{ }_{0} D_{t}^{\gamma} u-(1-2 \beta) \tau K \partial_{x}^{2} u\right],
\end{gathered}
$$

where $x_{r}$ is the location of the reagent $B$. In the case $\beta=1 / 2$, the obtained equation is reduced to that derived in the framework of the CTRW model.

\section{Models for activation-limited reactions}

\subsection{Linear reaction rates}

Another class of models, which is appropriate for activation limited reactions, is based on the assumption that the instantaneous reaction rate is unaffected by the diffusion factors. Since the subdiffusion process depends on the whole history of the system evolution, due to the memory effect, and the composition of the mixture changes with time, the diffusion and reaction are intertwined. The corresponding subdiffusionreaction equations have been first derived by Sokolov et al. [21] for the simplest linear reaction, when the density $u$ of the component $A$ evolves according to the equation

$$
\frac{d u}{d t}=-r u
$$

(similar to that of a radioactive decay) in the spatially homogeneous case. A simplistic subdiffusionreaction equation

$$
\partial_{t} u=\partial_{x}^{2}\left(K_{0} D_{t}^{1-\gamma} u\right)-r u
$$

is incorrect; it leads to unphysical negative values of the density $u$ in finite time [17]. Instead, one has to use integro-differential master equations for evolution of densities of chemical components (see, e.g., 
[37] and references therein). The predictions of those models are quite different from the predictions of models described in Sec. 2 (see, e.g., [38]).

In some limits, integro-differential equations can be reduced to PDEs. In the case of reaction (3.1), the corresponding PDE is as follows:

$$
\partial_{t} u=\partial_{x}^{2}\left[K e^{-r t} D_{t}^{1-\gamma}\left(e^{r t} u\right)\right]-r u
$$

$[21],[22],[27]$. In a multicomponent system, in the case where the evolution of the spatially homogeneous system is governed by the linear equation

$$
\frac{d \mathbf{u}}{d t}=\mathbf{M u},
$$

where $\mathrm{M}$ is a constant matrix, the following subdiffusion-reaction equation can be derived from the CTRW model [22],

$$
\partial_{t} \mathbf{u}=\partial_{x}^{2}\left[e^{\mathrm{M}_{t}} \mathrm{~K}_{0} D_{t}^{1-\gamma}\left(e^{-\mathrm{M} t} \mathbf{u}\right)\right]+\mathrm{Mu},
$$

where $\mathrm{K}$ is the matrix of diffusion coefficients.

A subdiffusion-reaction equation of this kind can be derived also in the case when $r$ is a function of $x$ [39], [40]. In that case, equation (3.3) is rewritten as

$$
\partial_{t} u(x, t)=\partial_{x}^{2}\left[K e^{-r(x) t} D_{t}^{1-\gamma}\left(e^{r(x) t} u(x, t)\right)\right]-r u(x, t) .
$$

An additional term $R^{+}(x, t)$ has to be added to the right-hand side of (3.6) if the reaction creates some new particles [41].

A further generalization of the subdiffusion-reaction equation in the case of spatio-temporal inhomogeneities of the particle creation function, $R^{+}(x, t)$, particle destruction rate, $r^{-}(x, t)$, and external forcing, $F(x, t)$, as well as a spatial inhomogeneity of the waiting time distribution, determined by the relations

$$
w(x, t)=0, t \in[0, \tau) ; w(x, t)=\frac{\gamma(x) \tau^{\gamma(x)}}{t^{1+\gamma(x)}}, t \in[\tau, \infty) ; 0<\gamma(x)<1,
$$

has been obtained in the framework of the CTRW model in [42]:

$$
\begin{gathered}
\left.\partial_{t} u(x, t)=\partial_{x}^{2}\left[K(\gamma(x)) \theta(x, t)_{0} D_{t}^{1-\gamma(x)}\left(\frac{u(x, t)}{\theta(x, t)}\right)\right]-\partial_{x}\left[\zeta^{-1}(\gamma(x)) F(x, t)\right) \theta(x, t)_{0} D_{t}^{1-\gamma(x)}\left(\frac{u(x, t)}{\theta(x, t)}\right)\right] \\
-r^{-}(x, t) u(x, t)+R^{+}(x, t),
\end{gathered}
$$

where $u(x, t)$ is the particle density,

$$
\theta(x, t)=\exp \left[-\int_{0}^{t} r^{-}\left(x, t^{\prime}\right) d t^{\prime}\right]
$$

is the survival probability for particle in the point $x$ from the beginning of the process to the time $t$, $K(\gamma(x))$ and $\zeta(\gamma(x))$ are some finite and differentiable functions. It should be noted that in the absence of reaction, the spatial inhomogeneity of the parameter $\gamma(x)$ leads to the accumulation of particles in the point with the minimum value of $\gamma(x)$ [43], i.e., the Gibbs-Bolzmann distribution is never achieved. However, as it is shown in [44], the random "death" of particles renormalizes diffusion and drift coefficients in the Fokker-Planck equation and restores the robustness of the model with respect to spatial fluctuations of the anomaly exponent $\gamma$. A fractional Fokker-Planck equation with varying anomaly exponent has been studied in a biological context in [45]. 


\subsection{Nonlinear reaction rates}

In the case of a nonlinear dependence of reaction rates on reactant densities, some generalizations of equations (3.5) have been suggested. Froemberg et al. [46] have considered the $A+B \rightarrow 2 A$ reaction under subdiffusion characterized by a waiting time pdf, $w(t)$. An integro-differential equation,

$$
\partial_{t} u(x, t)=\partial_{x}^{2} \int_{0}^{t} K(t-\tau) u(x, \tau) \exp \left[-\int_{\tau}^{t} k\left[1-u\left(x, \tau^{\prime}\right)\right] d \tau^{\prime}\right] d \tau-k[1-u(x, t)] u(x, t),
$$

has been obtained. Here the Laplace transform of the kernel, $\tilde{K}(s)$, is proportional to $s \tilde{w}(s) /[1-\tilde{w}(s)]$, where $\tilde{w}(s)$ is the Laplace transform of the waiting time pdf. A number of nonlinear subdiffusion-reaction models have been derived by Fedotov [47]. The nonlinearity of the reaction term in the form $R(u)=r(u) u$ can be taken into account by the following modification of equation (3.3):

$$
\partial_{t} u=\partial_{x}^{2}\left[K \exp \left(\int_{0}^{t} r(u(x, \tau)) d \tau\right){ }_{0} D_{t}^{1-\gamma}\left(u(x, t) \exp \left(-\int_{0}^{t} r(u(x, \tau)) d \tau\right)\right)\right]+R(u) u .
$$

In the case of "rejuvenation" of particles after the chemical reaction [23], it is necessary to distinguish between the "birth" rate, $r^{+}(u)$, and the "death" rate, $r^{-}(u)$, which leads to the following integrodifferential equation,

$$
\begin{aligned}
\partial_{t} u(x, t)= & \int_{0}^{t} K(t-\tau)\left[\int_{-\infty}^{\infty} u(x-z, \tau) \exp \left(-\int_{\tau}^{t} r^{-}\left(u\left(x-z, \tau^{\prime}\right)\right) d \tau^{\prime}\right) w(z) d z-\right. \\
& \left.u(x, \tau) \exp \left(-\int_{\tau}^{t} r^{-}\left(u\left(x, \tau^{\prime}\right)\right) d \tau^{\prime}\right)\right] d \tau+r^{+}(u) u-r^{-}(u) u .
\end{aligned}
$$

In the case of nonlinear dependence of reaction rates on reactant densities, it is typically impossible to write a closed system of equations for concentrations $u_{i}(x, t)$. Since the probability of a molecule to perform a jump depends on the time passed since the molecule's last jump (the "age" of the molecule), according to the waiting time distribution, it is necessary to take into account the molecule age and to describe the system by the densities $n_{i}\left(x, t, t^{\prime}\right)$ of molecules that performed their last jump to the point $x$ at the time instant $t^{\prime}$ and still reside at that point at the time instant $t$ (the molecule age $\tau=t-t^{\prime}$ is the argument of the waiting time probability distribution function). As mentioned in the introduction, a somewhat controversial point is the age of the molecule transformed into a molecule of another kind due to a chemical reaction without jumping. If the waiting time distribution for jumps is determined by the probability distribution of fluctuations in the environment that make the molecule release possible, it is natural to assume that the age of the molecule, understood as the argument of the waiting time pdf, is not changed due to the participation of the molecule in the reaction [21], [27]. In the latter case, the evolution equation for densities can be written [34] as

$$
\partial_{t} n_{i}\left(x, t, t^{\prime}\right)=-W_{i}\left(t-t^{\prime}\right) n_{i}\left(x, t, t^{\prime}\right)+\sum_{j=1}^{N} M_{i j}\left(u_{1}, \ldots, u_{N}\right) n_{j}\left(x, t, t^{\prime}\right), t>t^{\prime}
$$

where $n_{i}\left(x, t, t^{\prime}\right)$ is the density of particles of the type $i$ at the time instant $t$ that performed their last jump to the point $x$ at the time instant $t^{\prime}, W_{i}(\tau)$ is the jump rate function related to the waiting time distribution $w_{i}(\tau)$ by

$$
W_{i}(\tau)=\frac{w_{i}(\tau)}{1-\int_{0}^{\tau} w_{i}\left(\tau^{\prime}\right) d \tau^{\prime}}
$$

and

$$
u_{i}(x, t)=\int_{0^{-}}^{t} n_{i}\left(x, t, t^{\prime}\right) d t^{\prime}, t>0
$$


is the total density of the component $i$. If the waiting time distributions have the form

$$
w_{i}(\tau)=\frac{\gamma \tau_{i}^{\gamma}}{\left(\tau+\tau_{i}\right)^{\gamma+1}},
$$

then the functions $W_{i}(\tau)$ are

$$
W_{i}(\tau)=\frac{\gamma}{\tau+\tau_{0}}, 0<\gamma<1 .
$$

The first term on the right-hand side of (3.12) corresponds to the decrease of the density of particles that belong to a definite generation due to subdiffusion, and the second term describes its change due to the chemical reaction, which can be either negative or positive, unlike the models with "rejuvenation" of particles [23], [24], where it can be only negative. Equation (3.12) is supplemented by the boundary condition

$$
n_{i}(x, t, t)=\int_{\infty}^{\infty} m_{i}\left(x-x^{\prime}\right) d x^{\prime} \int_{0^{-}}^{t} W_{i}\left(t-t^{\prime}\right) n_{i}\left(x, t, t^{\prime}\right) d t^{\prime}+u_{0 i}(x) \delta(t),
$$

which describes the subdiffusion. Here $m_{i}$ is the jump length pdf for particles of the type $i$.

In the framework of system (3.12), (3.13), the "age structure" of the population of molecules depends on time and has a tendency of "aging", which leads to the decrease of the jump probability and hence slowing down of all the processes, including the front propagation ("propagation failure") [46], [30]. Specifically, if the jump rates of all the components coincide, $W_{i}(\tau)=W(\tau)$ for all $i$, in the large scale limit the problem is reduced to a normal diffusion-reaction system with the matrix of the diffusion coefficients decaying like $t^{\gamma-1}$ [34]. That leads to a decrease of the front velocity $c \sim t^{-\alpha}$ and of the front thickness $\delta \sim t^{-\alpha}$, where $\alpha=(1-\gamma) / 2$.

Another approach is adopted in [23], [24] where it is assumed that a molecule born in the course of reaction has zero age, as if it had arrived at the reaction point from somewhere else: the argument of the waiting time destribution is set to zero after the chemical transformation of the molecule. In that case, equation (3.12) includes only reactions leading to the "death" of molecules, which are assumed to be independent of the particle age, thus

$$
\partial_{t} n_{i}\left(x, t, t^{\prime}\right)=-W_{i}\left(t-t^{\prime}\right) n_{i}\left(x, t, t^{\prime}\right)-\frac{R_{i}^{-}\left(u_{1}, \ldots, u_{N}\right)}{u_{i}(x, t)} n_{i}\left(x, t, t^{\prime}\right)
$$

The "birth" processes appear in the boundary conditions (3.13):

$$
n_{i}(x, t, t)=\int_{\infty}^{\infty} d x^{\prime} \int_{0}^{t} \lambda\left(x-x^{\prime}, t-t^{\prime}\right) n_{i}\left(x, t, t^{\prime}\right) d t^{\prime}+R_{i}^{+}\left(u_{1}, \ldots, u_{N}\right),
$$

where $\lambda\left(x-x^{\prime}, t-t^{\prime}\right)$ is the rate of jumps from $x^{\prime}$ to $x$ for particles of the age $t-t^{\prime}$, hence

$$
W_{i}\left(t-t^{\prime}\right)=\int_{-\infty}^{\infty} \lambda\left(\xi, t-t^{\prime}\right) d \xi
$$

Here $R_{i}^{-} \geq 0$ and $R_{i}^{+} \geq 0$ are the death and birth reaction functions, correspondingly.

Due to the "rejuvenation", the molecule population achieves a stationary age distribution, and hence the reaction front velocity tends to a constant value [26].

For any particular natural problem, the applicability of each of the assumption has to be validated through a microscopic probabilistic theory or an experiment.

\section{Instabilities and pattern formation}

The analysis of instabilities in subdiffusion-reaction systems has some specific features. For normal diffusion-reaction systems, the standard linear stability analysis of a time-independent, spatially homogeneous base solution $\left\{u_{i}^{0}\right\}, i=1, \ldots, N$ is the search for solutions $\Delta u_{i}(x) \exp (\lambda t)$ of a linearized equation. 
If at least one of the eigenvalues $\lambda$ has a positive real part, the base solution is unstable. In the case of a subdiffusion-reaction system, that approach cannot be directly applied, because $\exp (\lambda t)$ is just not an eigenfunction of the fractional derivative operators, ${ }_{a} D_{t}^{\alpha}$ or ${ }_{a}^{C} D_{t}^{\alpha}$. The correct approach is to solve the linearized equation with arbitrary initial condition at $t=0,\left\{\Delta u_{i}(x, 0)\right\}$ and to consider the asymptotic behavior of the solution $\left\{\Delta u_{i}(x, t)\right\}$ at large $t$. That problem is normally solved by the Laplace transform $\Delta u_{i}(x, t) \rightarrow \Delta \tilde{u}_{i}(x, s)$. It turns out [48] that $\Delta \tilde{u}_{i}(x, s)$ have pole singularities at the values of $s$ that can be found formally by substitution of $\Delta u_{i}(x) \exp (s t)$ into the equations and the replacement of ${ }_{a} D_{t}^{\alpha} \exp (s t)$ and ${ }_{a}^{C} D_{t}^{\alpha} \exp (s t)$ with $s^{\alpha} \exp (s t)$. When the inverse Laplace transform is carried out by means of the integration along the Bromwich contour, these poles give exponential contributions into the large-time asymptotics of $\left\{\Delta u_{i}(x, t)\right\}$. It should be emphasized that not all of the poles with $\operatorname{Re} s>0$ create an instability. That surprising circumstance, generally known in the theory of fractional differential equations [49], was first demonstrated in the context of subdiffusion-reaction equations by Henry and Wearne [48] who investigated a pair of equations with $\gamma$. It has been shown that for $z=s^{1 / 2}$, only the poles that have both $\operatorname{Re} z>0$ and $\operatorname{Re} z^{2}>0$, indeed generate an asymptotically exponential growth of disturbances. In the general case, the instability takes place if $\Delta \tilde{u}_{i}(x, s)$ have a pole at $s=|s| \exp (i \phi)$ such that

$$
-\frac{\pi \gamma}{2} \leq \phi \leq \frac{\pi \gamma}{2}
$$

[50] - [54].

An instability of a stationary spatially-homogeneous state can lead either to a transition of the system to another stationary spatially-homogeneous state, or to the development of spatially-inhomogeneous or/and time-dependent patterns. In the former case, the propagation of fronts separating domains of different phases has the crucial role. In the present paper, we do not consider that scenario of the instability development relegating the reader to a recent review paper [55].

In the case of a short-wave instability, where the most unstable mode has a wavenumber $k_{c} \neq 0$, one can expect the development of spatially-inhomogeneous stationary (Turing) or oscillatory patterns. The early investigations of the Turing instability in subdiffusion-reaction systems [48], [56], [50] have been carried out for systems of equations of the type of (3.2); both monotonic and oscillatory modes of instability have been revealed. In [52], the short-wave instability for a two-species activator-inhibitor system with arbitrary powers of fractional operators acting in diffusion and reaction terms has been studied. It has been found that in the case of the Seki-Lindenberg model, (2.1), the oscillatory mode of a short-wave instability disappears. Note, that a short-wave oscillatory instability has been revealed for that model outside the region of its physical relevance for subdiffusion-limited reactions, when $\gamma>\gamma_{0}>1$ [51], [53], [57], [58].

A linear analysis of Turing instability in the case of activation-limited reactions (see Sec. 3) has been carried out in [28], in the spirit of [21]. In the case when the homogeneous system is governed by the system of equations

$$
\frac{d u_{i}}{d t}=f_{i}\left(u_{1}, \ldots, u_{N}\right), i=1, \ldots, N
$$

the subdiffusion is governed by the equation

$$
\Delta u_{i}(\mathbf{x}, t)=\int_{\Omega} \int_{0}^{t} m_{i}\left(\mathbf{x}-\mathbf{x}^{\prime}\right) w\left(t-t^{\prime}\right) \sum_{j=1}^{n}\left(e^{\nabla \mathbf{f}\left(t-t^{\prime}\right)}\right)_{i j} \Delta u_{j}\left(\mathbf{x}^{\prime}, t^{\prime}\right) d t^{\prime} d \mathbf{x}^{\prime},
$$

where

$$
(\nabla \mathbf{f})_{i j}=\frac{\partial f_{i}}{\partial u_{j}}
$$

(the derivatives are calculated in the point corresponding to the stationary homogeneous base state). It was shown that only a monotonic instability is possible for finite wavenumbers.

A weakly nonlinear analysis of the development of instabilities in subdiffusion-reaction systems has been performed in [25] in the framework of model (3.12), (3.13) with equal jump rate functions of the 
components, $w_{i}(\tau)=w(\tau)$ for all $i$. In the case of a short-wave Turing instability, the Ginzburg-Landau equation with real coefficients has been derived for an envelope amplitude function. In the case of a long-wave Hopf instability, the complex Ginzburg-Landau equation has been obtained. Thus, despite the memory effects and a qualitatively different form of the governing nonlinear equation, the weakly nonlinear development of instability in the case of subdiffusion is quite similar to that known for normal diffusion-reaction systems.

In conclusion to this section, let us mention the works where the stability of spatially inhomogeneous structures in subdiffusive systems has been studied. In [59], dynamics and stability of spike-type solutions have been studied for a subdiffusive system with Gierer-Meinhardt kinetics. Oscillatory instabilities of spikes and multi-spike patterns have been revealed. An extended class of subdiffusion-reaction models has been investigated in [54]. A number of exact results concerning the stability of spikes has been obtained. The stability and slow dynamics of two-spike patterns has been studied in [60].

\section{Applications}

In the present section, we list some applications of subdiffusion-reaction models to particular natural phenomena.

In some of the applications, models with fractional derivatives of the kind (2.2) are used. For instance, Kosztołowicz and Lewandowska [33] have applied that kind of equations for modelling the formation of a carious lesion of the tooth enamel. The theoretically predicted scaling law $x_{r}(t) \sim t^{\gamma / 2}$ for the location of the reaction zone center has been used for finding the parameter $\gamma$ from the experimental data. Henry et al. [61] have compared the predictions of a fractional diffusion model for spiny neuronal dendrites derived from CTRW with those of a model related to FBM, which was based on a usual reaction-diffusion equation with time-dependent coefficients.

Fedotov and Iomin [62], [63] have applied integro-differential equations for modelling a tumor cell spreading. The cells in state 1 (migratory phenotype) spread in a subdiffusive way but do not multiply, while the cells in state 2 (proliferating phenotype) multiply but do not migrate. The cells change their phenotypes with definite switching rates.

In a number of papers, fractional models with intertwined diffusion and reaction have been used. In [41], the phenomenon of fluorescence recovery after photobleaching (FRAP) has been considered as the reaction $F+S \leftrightarrow C$, where $F$ represents free proteins subjected to subdiffusion, $S$ denotes abundant vacant binding sites, and $C$ represents motionless bound complexes. That leads to equation (3.3) for the concentration of free proteins coupled with the reaction equation for the concentration of bound proteins. Model (3.6) has been applied in [40] for studying the morphogen gradient formation, which is crucial for embryonic cell development. The same problem has been tackled in [64] using a generalization of model (3.10) with the spatially inhomogeneous diffusion coefficient $K(x)$ and anomaly exponent $\gamma(x)$.

Acknowledgements. The work has been partially supported by the Israel Science Foundation (grant no. 680/10).

\section{References}

[1] J.-P. Bouchaud, A. Georges. Anomalous diffusion in disordered media: statistical mechanisms, models and physical applications. Phys. Rep., 195 (1990), 127-293.

[2] D. ben-Abraham, S. Havlin. Diffusion and Reactions in Fractals and Disordered Systems. Cambridge University Press, 2000 .

[3] R. Metzler, J. Klafter. The random walk's guide to anomalous diffusion: a fractional dynamics approach. Phys. Rep., 339 (2000), 1-77.

[4] R. Metzler, J. Klafter. The restaurant at the end of the random walk; recent developments in the description of anomalous transport by fractional dynamics. J. Phys. A: Math. Gen., 37 (2004), R161-R208.

[5] W. Feller. An Introduction to Probability Theory and Its Applications, v. I. John Wiley \& Sons, New York et al., 1968.

[6] W. Feller. An Introduction to Probability Theory and Its Applications, v. II. John Wiley \& Sons, New York et al., 1971. 
[7] B. B. Mandelbrot, J. W. van Ness. Fractional Brownian motions, fractional noises and applications. SIAM Rev., 10 (1968), 422-437.

[8] I. Goychuk, P. Hänngi. Anomalous escape governed by the thermal 1/f noise. Phys. Rev. Lett., 99 (2007), 200601.

[9] I. Goychuk. Viscoelastic subdiffusion: generalized Langevin equation approach. Adv. Chem. Phys., 150 (2012), $187-253$.

[10] A. Taloni, A. Chechkin, J. Klafter. Generalized elastic model: fractional Langevin description, fluctuation relation and linear response. Math. Model. Nat. Phenomen., 8 (2) (2013), 127-143.

[11] Y. Meroz, I. M. Sokolov, J. Klafter. Subdiffusion of mixed origin: When ergodicity and nonergodicity coexist. Phys. Rev. E, 81 (2010), 010101(R).

[12] Y. Meroz, I. M. Sokolov, J. Klafter. Unequal twins: probability distributions do not determine everything. Phys. Rev. Lett., 107 (2011), 260601.

[13] Y. Meroz, I. M. Sokolov. A toolbox for determining subdiffusive mechanisms. Phys. Rep., 573 (2015) 1-29.

[14] Y. Meroz, I. M. Sokolov, J. Klafter. Test for determining a subdiffusive model in ergodic systems from dingle trajectories. Phys. Rev. Lett., 110 (2013), 090601.

[15] D. H. Zanette. Wave fronts in bistable reactions with anomalous Lévy-flight diffusion. Phys. Rev. E, 55 (1997) 11811184.

[16] D. del-Castillo-Negrete. Truncation effects in superdiffusive front propagation with Lévy flights. Phys. Rev. E, 79 (2009), 031120.

[17] B. I. Henry, T. A. M. Langlands, S. L. Wearne. Anomalous diffusion with linear reaction dynamics: From continuous time random walks to fractional reaction-diffusion equation. Phys. Rev. E, 74 (2006), 031116.

[18] K. Seki, M. Wojcik, M. Tachiya. Fractional reaction-diffusion equation. J. Chem. Phys., 119 (2003), 2165-2170.

[19] K. Seki, M. Wojcik, M. Tachiya. Recombination kinetics in subdiffusive media. J. Chem. Phys., 119 (2003), $7525-7533$.

[20] S. B. Yuste, L. Acedo, K. Lindenberg. Reaction front in an $A+B \rightarrow C$ reaction-subdiffusion process. Phys. Rev. E, 69 (2004), 036126.

[21] I. M. Sokolov, M. G. W. Schmidt, F. Sagués. Reaction-subdiffusion equations. Phys. Rev. E, 73 (2006), 031102.

[22] T. A. M. Langlands, B. I. Henry, S. L. Wearne. Anomalous subdiffusion with multispecies linear reaction dynamics. Phys. Rev. E, 77 (2008), 021111.

[23] M. O. Vlad, J. Ross. Systematic derivaton of reaction-diffusion equations with distributed delays and relations to fractional reaction-diffusion equations: application to the theory of neolithic transition. Phys. Rev. E, 66 (2002), 061908.

[24] A. Yadav, W. Horsthemke. Kinetic equations for reaction-subdiffusion systems: derivation and stability analysis. Phys. Rev. E, 74 (2006), 066118.

[25] Y. Nec, A. A. Nepomnyashchy. Amplitude equations for a sub-diffusive reaction-diffusion system. J. Phys. A: Math. Theor., 41 (2008), 385101.

[26] V. Méndez, S. Fedotov, W. Horsthemke. Reaction-Transport Systems. Springer, Berlin, 2010.

[27] M. G. W. Schmidt, F. Sagués, I. M. Sokolov. Mesoscopic description of reactions for anomalous diffusion: a case study. J. Phys.: Condens. Matter, 19 (2007), 065118.

[28] Y. Nec, A. A. Nepomnyashchy. Turing instability in sub-diffusive reaction-diffusion systems. J. Phys. A: Math. Theor., 40 (2007), 14687-14702.

[29] D. Campos, V. Méndez. Nonuniversality and the role of tails in reaction-subdiffusion fronts. Phys. Rev. E, 80 (2009), 021133.

[30] H. H. Schmidt-Martens, D. Froemberg, I. M. Sokolov. F. Sagués. Front propagation in a one-dimensional autocatalytic reaction-subdiffusion system. Phys. Rev. E, 79 (2009), 041135.

[31] T. Kosztołowicz, K. D. Lewandowska. Application of fractional differential equations in modelling the subdiffusionreaction process. Math. Model. Nat. Phenom., 8 (2) (2013), 44-54.

[32] T. Kosztołowicz, K. D. Lewandowska. Time evolution of the reaction front in a subdiffusive system. Phys. Rev. E, 78 (2008), 066103.

[33] T. Kosztołowicz, K. D. Lewandowska. Time evolution of the reaction front in the system with one static and one subdiffusive component. Acta Phys. Polon. B, 37 (2006), 1571-1577.

[34] Y. Nec, V. A. Volpert, A. A. Nepomnyashchy. Front propagation problems with subdiffusion. Discr. Cont. Dyn. Syst., 27 (2010), 827-846.

[35] T. Kosztołowicz. Cattaneo-type subdiffusion-reaction equation. Phys. Rev. E, 90 (2014), 042151.

[36] A. A. Nepomnyashchy, V. A. Volpert. An exactly solvable model of subdiffusion-reaction front propagation. J. Phys. A: Math. Theor., 46 (2013), 065101.

[37] S. K. Hansen, B. Berkowitz. Integrodifferential formulations of the continuous-time random walk for solute transport subject to biomolecular $A+B \rightarrow 0$ reactions: From micro- to mesoscopic. Phys. Rev. E, 91 (2015), 032113.

[38] D. Froemberg, I. M. Sokolov. Stationary fronts in an $A+B \rightarrow 0$ reaction under subdiffusion. Phys. Rev. Lett. 100 (2008), 108304.

[39] K. Seki, A. I. Shushin, M. Wojcik, M. Tachiya. Specific features of the kinetics of fractional-diffusion assisted geminate reactions. J. Phys.: Condens. Matter 19 (2007), 065117.

[40] S. B. Yuste, E. Abad, K. Lindenberg. Reaction-subdiffusion model of morphogen gradient formation. Phys. Rev. E, 82 (2010), 061123.

[41] S. B. Yuste, E. Abad, K. Lindenberg. A reaction-subdiffusion model of fluorescence recovery after photobleaching (FRAP). J. Stat. Mech. - Theory and Exp., (2014), P11014. 
[42] C. N. Angstmann, I. C. Donelly, B. I. Henry. Continuous time random walk with reactions, forcing and trapping. Math. Model. Nat. Phenom., 8 (2) (2013), 17-27.

[43] S. Fedotov, S. Falconer. Subdiffusive master equation with space-dependent anomalous exponent and structural instability. Phys. Rev. E, 85 (2012), 031132.

[44] S. Fedotov, S. Falconer. Random death process for the regularization of subdiffusive fractional equations. Phys. Rev. E, 87 (2013), 052139.

[45] S. Fedotov, A. O. Ivanov, A. Y. Zubarev. Non-homogeneous random walks, subdiffusive migration of cells and anomalous chemotaxis. Math. Model. Nat. Phenom. 8 (2) (2013), 28-43.

[46] D. Froemberg, H. Schmidt-Martens, I. M. Sokolov, F. Sagués. Front propagation in $A+B \rightarrow 2 A$ reaction under subdiffusion. Phys. Rev. E, 78 (2008), 011128.

[47] S. Fedotov. Non-Markovian random walks and nonlinear reactions: subdiffusion and propagating fronts. Phys. Rev. E, 81 (2010), 011117.

[48] B. I. Henry, S. L. Wearne. Existence of Turing instabilities in a two-species fractional reaction-diffusion system. SIAM J. Appl. Math., 62 (2002), 870-887.

[49] D. Matignon. Stability results for fractional differential equations with applications to control processing. Comput. Eng. Syst. Appl., 2 (1996), 963-970.

[50] Y. Nec, A. A. Nepomnyashchy. Linear stability of fractional reaction-diffusion systems. Math. Model. Nat. Phenom., 2 (2) (2007), 77-105.

[51] V.V. Gafiychuk, B.Y. Datsko. Stability analysis and oscillatory structures in time-fractional reaction-diffusion systems. Phys. Rev. E 75 (2007), 055201(R).

[52] Y. Nec, A. A. Nepomnyashchy. Turing instability of anomalous reaction - anomalous diffusion systems. Euro. J. Appl. Math., 19 (2008), 329-349.

[53] V. Gafiychuk, B. Datsko, V. Meleshko. Mathematical modeling of the time fractional reaction-diffusion systems. J. Comp. Appl. Math., 220 (2008), 215-225.

[54] Y. Nec, M. J. Ward. An explicitly solvable nonlocal eigenvalue problem and the stability of a spike for a sub-diffusive reaction-diffusion systems. Math. Model. Nat. Phenom., 8 (2) (2013), 55-87.

[55] V. A. Volpert, Y. Nec, A. A. Nepomnyashchy. Fronts in anomalous diffusion-reaction systems. Philos. Trans. R. Soc. Lond., A 371 (2013), 20120179.

[56] B. I. Henry, T. A. M. Langlands, S. L. Wearne. Turing pattern formation in fractional activator-inhibitor systems. Phys. Rev. E, 72 (2005), 026101.

[57] V. Gafiychuk, B. Datsko. Mathematical modeling of different types of instabilities in time fractional reaction-diffusion systems. Comp. Math. Appl., 59 (2010), 1101-1107.

[58] V. Gafiychuk, B. Datsko. Different types of instabilities and complex dynamics in reaction-diffusion systems with fractional derivatives. J. Comp. Nonlin. Dyn. 7 (2012), 031001.

[59] Y. Nec, M. J. Ward. Dynamics and stability of spike-type solutions to a one dimensional Gierer-Meinhardt model with sub-diffusion. Physica D 241 (2012) 947-963.

[60] Y. Nec, M. J. Ward. The stability and slow dynamics of two-spike patterns for a class of reaction-diffusion system. Math. Model. Nat. Phenom., 8 (5) (2013), 206-232.

[61] B. I. Henry, T. A. M. Langlands, S. L. Wearne. Fractional cable models for spiny neuronal dendrites. Phys. Rev. Lett., 100 (2008), 128103.

[62] S. Fedotov, A. Iomin. Migration and proliferation dichotomy in tumor-cell invasion. Phys. Rev. Lett., 98 (2007), 118101.

[63] S. Fedotov, A. Iomin. Probabilistic approach to a proliferation and migration dichotomy in tumor cell invasion. Phys. Rev. E, 77(2008), 031911.

[64] S. Fedotov, S. Falconer. Nonlinear degradation-enhanced transport of morphogens performing subdiffusion. Phys. Rev. E, 89 (2014), 012107. 